\title{
LIMITS TO EXTENSIONS OF BURGERS' EQUATION
}

\author{
BY \\ STEVEN NERNEY (NASA-Marshall Space Flight Center, AL and Ohio University, Lancaster), \\ EDWARD J. SCHMAHL (Astronomy Department, University of Maryland, College Park, MD), \\ A.ND \\ Z. E. MUSIELAK (Dept. of Mech. and Aerospace Engineering, Univ. of Alabama at Huntsville)
}

\begin{abstract}
The vector Burgers' equation is extended to include pressure gradients and gravity. It is shown that within the framework of the Cole-Hopf transformation there are no physical solutions to this problem. This result is important because it clearly demonstrates that any extension of Burgers' equation to more interesting physical situations is strongly limited.
\end{abstract}

1. Introduction. We solved Burgers' equation in three dimensions for arbitrary orthogonal curvilinear coordinate systems in [1], hereafter referred to as NSM (also see [2]). The analytical solutions suffered from the well-known deficiencies of Burgers' equation, which does not account for gravity and pressure gradients, nor does it conserve mass. In this paper, we have attempted to extend Burgers' equation by including gravity and either isothermal or adiabatic pressure gradients. The basic idea of our approach is to split the momentum equation into the vector Burgers' equation and the equation that contains all remaining terms, and to solve these two equations simultaneously. We show that the latter can be done only when a special auxiliary condition is satisfied. Because the solutions to the vector Burgers' equation are well known [1], the main problem is to find physically acceptable solutions to the auxiliary condition that also satisfy the continuity equation. We have attempted to generate steady-state, viscous solutions by constructing them from the solution of Laplace's equation in such a way as to satisfy the continuity equation for compressible flow. Our apparent success is vitiated when we find that there are no physically acceptable solutions to the $2-\mathrm{D}$ viscous case that can be derived from the potential flow problem and produce physical pressure gradients. This is an important result because it shows that the obvious extension of the vector Burgers' equation to more interesting physical cases is strongly limited. Still, the solutions are of interest in the same sense that the analytic solutions of the vector Burgers' equation are interesting.

We begin our presentation by summarizing the assumptions and solutions of the vector Burgers' equation presented in paper [1], and then describe in detail our new results.

Received Narch 22, 1994.

1991 Mathematics Subject Classification. Primary 35K99.

(C) 1996 Brown University 
2. Basic equations. The vector Burgers' equation can be written in the following form:

$$
\frac{\partial \vec{u}}{\partial t}+\vec{u} \cdot \nabla \vec{u}=\nu \nabla^{2} \vec{u}
$$

We proved that the solution of this equation can be expressed as a function of the solution of the diffusion equation:

$$
\frac{\partial \Theta}{\partial t}=\nu \nabla^{2} \Theta
$$

namely,

$$
\vec{u}=-\frac{2 \nu}{\Theta} \nabla \Theta .
$$

This result is correct for arbitrary curvilinear coordinate systems and is a subset of the more general solutions to the tensor Burgers' equation [2]. We would like to be able to extend our previous solutions to include pressure gradients and gravity, as well as satisfying the continuity equation. Then we must solve the momentum equation,

$$
\frac{\partial \vec{u}}{\partial t}+\vec{u} \cdot \nabla \vec{u}=-\frac{\nabla P}{\rho}+\frac{\nabla G M}{r}+\nu \nabla^{2} \vec{u}+\vec{f}
$$

where $\vec{f}$ is a general conservative force per unit mass so that

$$
\vec{f}=\nabla \Phi_{f}
$$

and $\rho, P, G, M$ are density, pressure, the gravitational constant, and the mass of the object exerting the gravitational pull, respectively. The continuity equation is written as

$$
\frac{\partial \rho}{\partial t}+\nabla \cdot(\rho \vec{u})=0
$$

In order to evaluate the vector Laplacian for arbitrary orthogonal coordinate systems, we must use

$$
\nabla^{2} \vec{u}=\nabla(\nabla \cdot \vec{u})-\nabla \times(\nabla \times \vec{u})
$$

and for the inertial term

$$
\vec{u} \cdot \nabla \vec{u}=\nabla \frac{u^{2}}{2}-\vec{u} \times(\nabla \times \vec{u})
$$

Then Eq. (4) greatly simplifies as long as

$$
\vec{u} \times(\nabla \times \vec{u})=\nu \nabla \times(\nabla \times \vec{u})
$$

This appears to be an equation that represents quite general flows, but the only solutions are irrotational. This can be seen by taking the divergence of both sides of Eq. (9a) and using

$$
\nabla \cdot(\vec{A} \times \vec{B})=\vec{B} \cdot(\nabla \times \vec{A})-\vec{A} \cdot(\nabla \times \vec{B}) .
$$

Therefore, we require

$$
\nabla \times \vec{u}=0
$$


and

$$
\vec{u}=\nabla \Phi
$$

We also use

$$
\frac{\nabla P}{\rho}=\nabla\left(\int \frac{d P}{\rho}\right)
$$

so that all terms in Eq. (4) are the gradient of a scalar. The integral of the pressure term can be done whenever $P=P(\rho)$, but we will solve Eq. (4) in the limit of an isothermal pressure gradient with the ideal gas law $\left(P=a^{2} \rho\right.$, where $a$ is the isothermal sound speed). Then the pressure gradient term may be written as

$$
\frac{\nabla P}{\rho}=a^{2} \nabla \ln \rho
$$

Now, using Eqs. (9a), (11a), and (12), Eq. (4) simplifies to

$$
\nabla\left[\frac{\partial \Phi}{\partial t}+\frac{(\nabla \Phi)^{2}}{2}+a^{2} \ln \rho-\frac{G M}{r}-\nu \nabla^{2} \Phi-\Phi_{f}\right]=0 .
$$

This implies that the integral of Eq. (13) is only a function of time, so that

$$
\frac{\partial \Phi}{\partial t}+\frac{(\nabla \Phi)^{2}}{2}+a^{2} \ln \rho-\frac{G M}{r}-\nu \nabla^{2} \Phi-\Phi_{f}=E(t)
$$

The following vector identity is useful and leads to an important form for $\nabla^{2} \Phi$ :

$$
\nabla \alpha=\frac{\partial \alpha}{\partial \Theta} \nabla \Theta
$$

which is true in any orthogonal coordinate system. Taking the divergence of both sides with $\alpha=\Phi$ and using Eq. (15a) again with $\alpha=d \Phi / d \Theta$ yields

$$
\nabla^{2} \Phi=\frac{d^{2} \Phi}{d \Theta^{2}}(\nabla \Theta)^{2}+\frac{d \Phi}{d \Theta} \nabla^{2} \Theta
$$

which, again, is valid for any orthogonal curvilinear coordinate system. Substituting Eq. (15b) into (14), we get

$$
\frac{\partial \Phi}{\partial t}+\frac{(\nu \Phi)^{2}}{2}+a^{2} \ln \rho-\frac{G M}{r}-\Phi_{f}-\nu\left(\frac{d^{2} \Phi}{d \Theta^{2}}(\nabla \Phi)^{2}+\frac{d \Phi}{d \Theta} \nabla^{2} \Theta\right)=E(t) .
$$

Now Eq. (2) guarantees that the first and last terms on the left-hand side will cancel so that

$$
(\nabla \Theta)^{2}\left[\frac{1}{2}\left(\frac{d \Phi}{d \Theta}\right)^{2}-\nu \frac{d^{2} \Phi}{d \Theta^{2}}\right]+a^{2} \ln \rho-\frac{G M}{r}-\Phi_{f}=E(t) .
$$

An important aspect of our work in NSM was that the remaining terms in the equation similar to Eq. (16b) were proportional to $(\nabla \Theta)^{2}$ so that this factor cancelled out, leading 
to an integrable equation for $\partial \Phi / \partial \Theta$. It is clear that the same procedure will work here provided that the following auxiliary condition is satisfied:

$$
a^{2} \ln \rho-\frac{G M}{r}-\Phi_{f}-E(t)= \pm C^{2}(\nabla \Theta)^{2}
$$

It is easy to show that $C$ must be found from the solution of

$$
C^{2}(\Theta)=\nu \frac{d^{2} \Phi}{d \Theta^{2}}-\frac{1}{2}\left(\frac{d \Phi}{d \Theta}\right)^{2}
$$

but this is not particularly helpful. We choose to examine possible solutions for the case of constant $C$ partly because $C=0$ corresponds to the vector Burgers' equation solution discussed by NSM, and we are attempting to find physical extensions of the Burgers' equation solutions. We are also physically motivated by noticing that the particular choice of $C^{2}=-\frac{1}{2}$ in Eq. (17a) reduces the auxiliary condition to the Bernoulli equation which must be solved for the pressure distribution implied by a potential flow model (the steady limit of the diffusion equation). The difference is that the auxiliary condition is for compressible flow. Additionally, as we shall also show, the case of constant $C$ generalizes known velocity laws derived from the solution of Burgers' equation. It is for these reasons that we examine the solutions of the irrotational momentum equation subject to the constraint of Eq. (17a).

The differential equation that results from substituting Eq. (17a) into Eq. (16b) (the analog of Eq. (7a) in NSM) is

$$
\left(\frac{d \Phi}{d \Theta}\right)^{2}=2 \nu \frac{d^{2} \Phi}{d \Theta^{2}} \mp 2 C^{2} .
$$

We solve for $\partial \Phi / \partial \Theta$ and use

$$
\vec{u}=\nabla \Phi=\frac{d \Phi}{d \Theta} \nabla \Theta
$$

to find

$$
\begin{gathered}
\frac{d \Phi}{d \Theta}=\sqrt{2} C \tan \left(\frac{C}{2 \nu} \Theta+C_{1}\right), \\
\vec{u}=\sqrt{2} C \tan \left(\frac{C}{2 \nu} \Theta+C_{1}\right) \nabla \Theta
\end{gathered}
$$

where $C_{1}$ is a constant of integration. This solution corresponds to the minus sign in Eq. (18), while

$$
\vec{u}=\sqrt{2} C \tanh \left(\frac{C}{2 \nu} \Theta+C_{1}\right) \nabla \Theta
$$

corresponds to the positive sign. The first solution seems nonphysical because of the singularities, although solutions could be generated by restricting the argument between 0 and $\pi / 4$. But, as we shall show shortly, the existence of any physical solutions requires choosing the hyperbolic tangent solution. It is also known $[3,5]$ that the limit of Eq. 
(19d) with $\nabla \Theta=$ constant is the steady solution to the 1-D Cartesian Burgers' equation for an initial step function profile with a sharp but finite discontinuity in $u$ at $x=a$ which rounds off asymptotically in time to a steady-state shock layer profile of thickness $\nu / u_{0}$ :

$$
u=-u_{0} \tanh \left[\frac{u_{0}(x-a)}{2 \nu}\right] .
$$

The tangent form is also known to be a steady-state solution of the Cartesian Burgers' equation [5].

The obtained solution, Eq. (19d), leads to a mechanical energy equation, which is found by taking the dot product of Eq. (19d) with $\vec{u}$ and then eliminating $(\nabla \Theta)^{2}$ in Eq. (17a). This gives

$$
\frac{u^{2}}{2} \operatorname{coth}^{2}\left(\frac{C}{2 \nu} \Theta+C_{1}\right)-\frac{G M}{r}+a^{2} \ln \rho-\Phi_{f}=E(t) .
$$

Note that, although this equation is similar to Bernoulli's equation, Eq. (20) is for nonsteady, viscous flow. The hyperbolic tangent solution can be seen as the appropriate form because the internal energy/gram will decrease if the speed increases. The form of the mechanical energy equation is strongly reminiscent of nonlinear decay models [1]. A singular source of mass at the origin requires solutions for $\operatorname{coth} \Theta$ that begin at finite radius close to the source, but then the motion decays so that the kinetic energy/mass approaches zero as $t \rightarrow \infty$ when $E(t) \rightarrow 0$.

We must now impose mass conservation on this model, and, to this end, we find a useful form for the continuity equation. Using Eq. (11a) and further eliminating $\nabla \cdot \vec{u}$ by using Eqs. (2) and (15b) yields:

$$
\left(\frac{\partial}{\partial t}+\vec{u} \cdot \nabla\right) \ln \rho+\frac{d^{2} \Phi}{d \Theta^{2}}(\nabla \Theta)^{2}+\frac{1}{\nu} \frac{\partial \Phi}{\partial t}=0 .
$$

To evaluate the continuity equation, we use streamline coordinates

$$
\hat{e}_{s}=\frac{\vec{u}}{|\vec{u}|}
$$

3. Steady solutions. We further limit physical space to steady solutions so that Eq. (2) reduces to Laplace's equation. Then Eq. (19d) requires that $\nabla \Theta$ is zero perpendicular to streamlines and $\nabla \Theta$ is $\partial \Theta / \partial s$. The problem reduces to finding that subset of the solution of Laplace's equation that satisfies the auxiliary condition and conserves mass. Identifying $\nabla \Theta$ with a velocity derived from a potential flow solution, we find

$$
\begin{aligned}
& \nabla \cdot \vec{u}_{\text {pot }}=0 \\
& \nabla \cdot(\rho \vec{u})=0
\end{aligned}
$$

so that

$$
\rho \vec{u}=\rho_{\text {pot }} \vec{u}_{\text {pot }}+\nabla \times \vec{A} .
$$


The following details show that $\nabla \times \vec{A}$ is zero.

The steady continuity equation is derived from Eq. (21),

$$
u \frac{\partial}{\partial s} \ln \rho+\frac{d^{2} \Phi}{d \Theta^{2}}\left(\frac{\partial \Theta}{\partial s}\right)^{2}=0
$$

Dividing by $\partial \Theta / \partial s$ and using

$$
u=\frac{d \Phi}{d \Theta} \frac{\partial \Theta}{\partial s}
$$

(for solutions with nonzero velocities), Eq. (24) integrates exactly with respect to $\Theta$ to

$$
\rho \frac{d \Phi}{d \Theta}=k_{1},
$$

where $k_{1}$ might be a function of spatial coordinates. Substituting Eq. (26) back into (19a),

$$
\rho \vec{u}=k_{1} \nabla \Theta
$$

or, if the original solution of Laplace's equation is formulated in terms of the velocity,

$$
\rho \vec{u}=k_{1} \vec{u}_{\text {pot }},
$$

and $k_{1}$ is seen to be the original incompressible density for the potential flow solution, $\rho_{\text {pot }}$, i.e., a strict constant. An important aspect of this method is that the new steady, compressible solutions are created from the old potential flow (i.e., incompressible) solutions, provided that the boundary condition on the compressible solution is formulated in terms of the mass flux.

Substituting the potential flow speed back into Eq. (20) yields another form for the steady mechanical energy equation:

$$
a^{2} \ln \rho-\frac{G M}{r}-\Phi_{f}-C^{2} u_{\text {pot }}^{2}=E_{0} .
$$

Setting $\Phi_{f}$ equal to zero and choosing

$$
C^{2}=-1 / 2
$$

we get

$$
a^{2} \ln \rho-\frac{G M}{r}+\frac{u_{\mathrm{pot}}^{2}}{2}=E_{0}
$$

The auxiliary condition is now seen to be the Bernoulli equation that must be used to solve for the pressure distribution implied by the potential velocity. However, we use Eq. (19d) to solve for the next higher-order solution for the velocity related to this variable pressure distribution, but including viscosity.

With this value for $C$, we obtain

$$
\vec{u}=-\tanh \left(\frac{\Theta}{2 \nu}+C_{1}\right) \vec{u}_{\text {pot }}
$$


and

$$
\rho=\rho_{\text {pot }} \operatorname{coth}\left(\frac{\Theta}{2 \nu}+C_{1}\right)
$$

The last part of this solution is to solve for the subset of physical potential flow solutions. Setting gravity equal to zero and substituting Eq. (30b) into (28a), we find

$$
u_{\text {pot }}^{2}=2 E_{0}-2 a^{2} \ln \operatorname{coth}\left(\frac{\Theta}{2 \nu}+C_{1}\right) .
$$

Replacing the $\int d P / \rho$ with the adiabatic term, $(\gamma-1) P /(\gamma \rho)$, and substituting $\gamma=5 / 3$, and then using Eq. (26) gives the related equation for the adiabatic case:

$$
u_{\text {pot }}^{2}=2 E_{0}-5 b \operatorname{coth}^{2 / 3}\left(\frac{\Theta}{2 \nu}+C_{1}\right),
$$

where we have also used the adiabatic equation of state,

$$
P=b \rho^{\gamma} \text {. }
$$

The potential flow solutions are limited to those whose speed is constrained to obey Eqs. (31a, b).

We use complex variables to show that there are no physical solutions that simultaneously satisfy both Laplace's equation and the constraint on the speed, Eqs. (31a, b). Writing the complex potential, $F(z)$, as

$$
F(z)=\Theta(x, y)+i \Psi(x, y)
$$

with

$$
z=x+i y
$$

then

$$
W(z)=\frac{d F}{d z}=v_{x}-i v_{y}
$$

where $\Psi$ is the stream function, and $W(z)$ is the complex velocity. The utility of this approach is obvious because

$$
W W^{*}=v_{x}^{2}+v_{y}^{2}
$$

where $W^{*}$ is the complex conjugate of $W$, which allows the velocity components to be read from Eqs. (31a) or (31b). The method of complex variables requires that these velocity components must be derived from an analytic potential function. In the following, we demonstrate that this is not possible.

Consider the general form of Eqs. $(31 \mathrm{a}, \mathrm{b})$ :

$$
(\nabla \Theta)^{2}=f(\Theta)
$$

This equation implies that the gradient of $(\nabla \Theta)^{2}$ must be parallel to $\nabla \Theta$, or that the Jacobian of $\Theta$ and $(\nabla \Theta)^{2}$ must be zero. This is a necessary condition for solutions to Eqs. $(31 \mathrm{a}, \mathrm{b})$ to exist. The Jacobian simplifies (in Cartesian coordinates) to

$$
\left(v_{x}^{2}-v_{y}^{2}\right) \frac{\partial v_{x}}{\partial y}-2 v_{x} v_{y} \frac{\partial v_{x}}{\partial x}=0
$$


where we have also used the Cauchy-Riemann equations,

$$
\begin{aligned}
& v_{x}=\frac{\partial \Phi}{\partial x}=\frac{\partial \Psi}{\partial y} \\
& v_{y}=\frac{\partial \Phi}{\partial y}=-\frac{\partial \Psi}{\partial x}
\end{aligned}
$$

But Eq. (35) is the same as

$$
\operatorname{Im}\left(W^{* 2}(z) \frac{d W(z)}{d z}\right)=0
$$

One solution is the trivial case of zero derivative. The other is found by defining $G(z)$ to be the reciprocal of $W(z)$, so that Eq. (37a) may be written as

$$
\operatorname{Im}\left(\frac{d G / d z}{\left(G^{*} G\right)^{2}}\right)=0 .
$$

Because the denominator is real, we require, except at poles,

$$
\operatorname{Im}\left(\frac{d G(z)}{d z}\right)=0 .
$$

But Eqs. (36) then require

$$
\frac{d G(z)}{d z}=0
$$

so that

$$
W(z)=\frac{c}{z},
$$

where $c$ is a complex constant. This gives the solution

$$
\Theta=a_{1} \ln r+a_{2},
$$

together with the trivial solution

$$
\Theta=a_{3} x+a_{14} y+a_{5}
$$

where the $a_{i}$ are constants. Since Eqs. (31a) and (31b) are not of this form, there are, then, no two-dimensional solutions of the potential flow problem that satisfy the physical constraint on the speed. Since these are required for solving for physical viscous, compressible solutions through Eqs. (29) and (30a), there are no physical solutions of this model. This is an important result because it shows that the method of solving Burgers' equation based on the Cole-Hopf transformation cannot be used to solve a general (and physically more interesting) problem of a flow with gravity and pressure gradients. To sum up, substituting Eq. (26) into Eq. (16b) clearly indicates that $(\nabla \Theta)^{2}$ is a function of $\Theta$. But, we have shown that the only steady solutions for $\nabla \Theta$ are those generated from Eqs. (40a, b). Our Cole-Hopf extension of the solution of Burgers' equation requires that $F(\Theta)$ must be a function of $\operatorname{coth}\left(\Theta / 2 \nu+C_{1}\right)$, so that there are no physical solutions. 
4. Conclusions. We have extended the solutions to the vector Burgers' equation [1] by a method that splits the momentum equation into two pieces, each of which is satisfied simultaneously. This allows us to solve a subset of irrotational flow problems with pressure gradients and gravity. The steady limit, when combined with mass conservation, reduces to specifying a subset of potential flow solutions. These incompressible flows, in turn, allow the generation of viscous, compressible solutions that are related to the solutions of the vector Burgers' equation [1]. We then proved that the subset of two-dimensional potential flow solutions does not correspond to the requirements for physical flow derived from real pressure gradients. In this sense, the pressure gradients are derived from pseudo-densities. It seems unlikely that physical models could be generated by this method using either three spatial dimensions or time-dependent solutions. Still, these results are of interest in the same sense that the analytic solutions of the vector Burgers' equation are interesting.

Acknowledgments. ZEM acknowledges partial support of this work by the NSF under grant no. ATM-9119580. EJS acknowledges partial support of this work under grant no. NAG 2001 from NASA-Goddard Space Flight Center.

\section{REFERENCES}

[1] S. Nerney, E. J. Schmahl, and Z. E. Musielak, Analytic solutions of the vector Burgers' equation, Quart. Appl. Math. 54, 63-71 (1996)

[2] K. B. Wolf, L. Hlavaty, and S. Steinberg, Non-linear differential equations as invariants under group action on coset bundles: Burgers and Korteweg-de Vries equation families, J. Math. Anal. Appl. 114, 340 359 (1986)

[3] J. D. Cole, On a quasi-linear parabolic equation occurring in aerodynamics, Quart. Appl. Math. 9, $225-236(1951)$

[4] E. Hopf, The partial differential equation $u_{t}+u u_{x}=\mu u_{x x}$, Comm. Pure Appl. Math. 3, 201-230 $(1950)$

[5] E. R. Benton, Solutions illustrating the decay of dissipation layers in Burgers' nonlinear diffusion equation, Phys. Fluids 10, 21132119 (1967) 